\title{
Modeling of non-stationary accretion disks in X-ray novae A 0620-00 and GRS 1124-68 during outburst
}

\author{
V. F. Suleimanov ${ }^{1,2}$, G. V. Lipunova ${ }^{3}$, and N. I. Shakura ${ }^{3}$ \\ 1 Institute for Astronomy and Astrophysics, Kepler Center for Astro and Particle Physics, Eberhard Karls University, Sand 1, \\ 72076 Tübingen, Germany \\ e-mail: suleimanov@astro.uni-tuebingen.de \\ 2 Kazan State University, Kremlevskaja str., 18, Kazan 420008, Russia \\ 3 Sternberg Astronomical Institute, Moscow State University, Universitetskij pr., 13, 119992, Moscow, Russia \\ e-mail: [galja;shakura]@sai.msu.ru
}

Received 7 May 2008 / Accepted 7 September 2008

\begin{abstract}
Aims. We address the task of modeling soft X-ray and optical light curves of X-ray novae in the high/soft state.

Methods. The analytic model of viscous evolution of an externally truncated accretion $\alpha$-disk is used. Relativistic effects near a Kerr black hole and self-irradiation of an accretion disk are taken into account.

Results. The model is applied to the outbursts of X-ray nova Monocerotis 1975 (A 0620-00) and X-ray nova Muscae 1991 (GRS 1124-68). Comparison of observational data with the model yields constraints on the angular momentum (the Kerr parameter) of the black holes in A 0620-00 and GRS 1124-68: 0.3-0.6 and $\leq 0.4$, and on the viscosity parameter $\alpha$ of the disks: $0.7-0.95$ and $0.55-0.75$. We also conclude that the accretion disks should have an effective geometrical thickness $1.5-2$ times greater than the theoretical value of the distance between the photometric layers.
\end{abstract}

Key words. binaries: close - X-rays: binaries - stars: individual: Nova Mon 1975 - stars: individual: Nova Mus 1991

\section{Introduction}

X-ray novae are the low-mass X-ray close binary systems (LMXBs) with a relativistic component, a black hole, or a neutron star (see, e.g., Tanaka \& Shibazaki 1996; Esin et al. 2000; Cherepashchuk 2000). Much attention has been drawn to $\mathrm{X}$-ray novae because they provide black hole candidates with the most reliable mass determinations (Cherepashchuk 2000; McClintock \& Remillard 2003). The secondary component is a red dwarf, which nearly fills its Roche lobe, and transfer rates are estimated to be as low as $10^{-10}-10^{-12} M_{\odot} / \mathrm{yr}$ (Tanaka \& Shibazaki 1996). Once every several decades, the accretion rate onto the central object rises (up to $10^{-8} M_{\odot} / \mathrm{yr}$ ), and the system flares up as a Nova at all wavelengths.

In a significant number of X-ray novae the X-ray flux declines in an exponential fashion after the peak of an outburst, with a characteristic time of 30-40 days (see, e.g., McClintock \& Remillard 2003). The optical flux also decays exponentially but about two times more slowly. The X-ray emission of the majority of X-ray novae during an outburst can be interpreted as the sum of a hard power-law component and a soft component produced by a multi-temperature black body disk with a maximum temperature 0.5-1 keV (Tanaka \& Shibazaki 1996). As a rule, during the first several tens of days after the peak, the soft component dominates the power-law component (this stage is called the high/soft state). After 100-150 days the spectrum becomes harder as the power-law component prevails. An outburst usually lasts for several months.

Up to now the mechanism of the outbursts in X-ray novae has not been completely understood, but it most probably involves an instability of the outer disk regions
(King \& Ritter 1998; Cherepashchuk 2000). This instability, related to hydrogen ionization, was initially proposed to explain dwarf novae, in which accretion occurs onto a white dwarf (Meyer \& Meyer-Hofmeister 1981; Smak 1984; Cannizzo 1993). Subsequently, the concept was extended to X-ray novae. van Paradijs (1996) followed by King \& Ritter (1998) suggested that irradiation of the outer parts of the accretion disk by central $\mathrm{X}$-rays has a significant impact on the dynamics of the evolutionary cycle in X-ray novae. Such irradiation is low in the dwarf novae (Dubus et al. 2001; Lasota 2001).

To explain the spectral evolution of X-ray novae, a number of models have been proposed (see review by McClintock \& Remillard 2003). Esin et al. (1997) proposed a scenario involving a change of cooling mechanisms with decreasing accretion rate. According to their model, at the outburst peak and through the subsequent 100-150 days, the disk is geometrically thin and optically thick and embedded in a hot corona. As the accretion rate falls, the disk, begining from the center, gradually becomes advection-dominated, geometrically thick, and optically thin (Narayan et al. 1998). Using a succession of stationary models with different accretion rates, Esin et al. (1997, 2000) describe the X-ray and optical light curves and the spectral evolution of X-ray nova Muscae 1991 and X-ray nova Monocerotis 1975. To explain the observed X-ray-to-optical flux ratio they considered an irradiation of the disks and assumed that 80-90\% of the incident X-ray flux is thermalized in the disks, which are thicker than given by the standard theory.

Naturally, time-dependent phenomena in disks call for a dynamical model. Whereas a stationary hydrostatic approximation can be kept to describe the vertical structure of the disk, a nonstationary model should primarily address its radial structure. 
A theory of time-dependent accretion $\alpha$-disks in binary systems has been worked out by Lipunova \& Shakura (2000, hereafter LS00) and subsequently used to describe X-ray and optical light curves of X-ray nova Monocerotis 1975 and X-ray nova Muscae 1991 (Lipunova \& Shakura 2002, hereafter LS02). The model applies to the first several tens of days after the outburst peak, when the spectral state is high/soft, the hydrogen is ionized in the whole disk, and before the secondary maximum. The disk is considered to be externally truncated due to tidal interactions in a binary. Fitting the observational light curves of the two X-ray novae provided estimates of the $\alpha$-parameter and relationships between the black hole mass and the distance to the systems. Actually, a new method of estimating the $\alpha$-parameter of accretion disks was proposed, and the $\alpha$-parameter turned out to be rather high, $0.2-0.6$.

In the present work, we develop the model of LS02 further. We include the effect of self-irradiation of the disk and take into account the general relativistic effects on the radial structure of the disk and on light propagation near a Kerr black hole. The last effect increases the degree of irradiation remarkably compared to the plain space-time geometry, even for a non-rotating black hole (Suleimanov et al. 2007).

In Sect. 2 theoretical aspects of the model are considered. The modeling procedure and general features of the solution are described in Sect. 3. Application of the model to the X-ray novae A 0620-00 and GRS 1124-68 follows in Sect. 4.

\section{The model}

\subsection{Time-dependent accretion disk}

The theory of time-dependent accretion has been put forward in the work of Lyubarskij \& Shakura (1987) and developed for an $\alpha$-disk in a close binary system by LSO0.

A diffusion-type differential equation of non-stationary accretion is solved analytically assuming specific outer boundary conditions. The size of the disk is fixed due to tidal effects, and the accretion rate through the outer boundary is negligible compared with the rate through the inner boundary of the disk. The opacity coefficient is a power-law function of density $\rho$ and temperature $T$. Separate regimes with scattering and Kramers absorption are considered. The vertical structure is treated anaytically on the basis of a method proposed by Ketsaris \& Shakura (1998). As a result, the accretion rate at each radius is a powerlaw function of time and of some other parameters. In the case of Kramers opacity, it is given by (LSO0):

$\dot{M}(R, t)=2 \pi \frac{1.224 y(\xi)}{h_{0}}\left(\frac{h_{0}^{14 / 5}}{D(t+\delta t)}\right)^{10 / 3}$.

Here $h_{0}=\sqrt{G M_{\mathrm{bh}} R_{\mathrm{out}}}$ is the specific angular momentum of the disk matter at the outer radius; $\delta t$ has dimensions of seconds and normalizes the initial accretion rate; $D$ is a dimensional "diffusion constant" depending on the $\alpha$-parameter, the black hole mass, and the opacity coefficient $\kappa_{0}$; function $y(\xi)$ is a polynomial truncated to three terms:

$y(\xi)=1.43-1.61 \xi^{5 / 2}+0.18 \xi^{5}$,

where $\xi=\sqrt{R / R_{\text {out }}}$ is the ratio of the angular momentum at a given disk radius to the angular momentum at the outer disk radius.

One can infer from (1) that the accretion rate near the central object hardly depends on $R$. The disk dynamics (variations

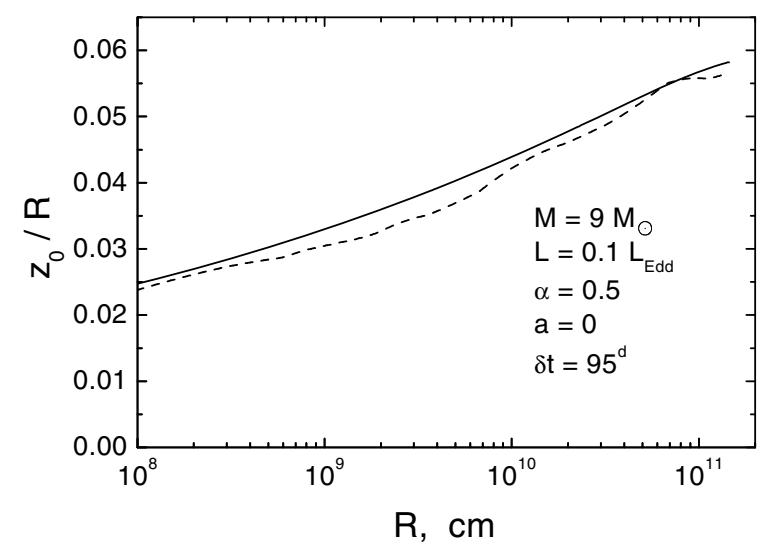

Fig. 1. Relative half-thickness of the disk calculated for Kramers opacity (solid line) and for the tabulated values of opacity (dashed line). The pertubations in the dashed line are caused by opacity variations.

in the accretion rate, in particular) are defined by its structure at outer radii, because it is there that the characteristic viscous timescale is the longest. Changes in the accretion rate, occurring on a smaller time scale at inner radii, respond to the course of evolution at the outer disk. Thus, to analyze the light curves of a fully ionized accretion disk in any spectral band, one can use solution (1) written for the case of Kramers opacity $\kappa=\kappa_{0} \rho T^{-7 / 2}$.

We take $\kappa_{0}=5 \times 10^{24} \mathrm{~cm}^{5} \mathrm{~K}^{7 / 2} \mathrm{~g}^{-2}$ (Frank et al. 2002, Chap. 5), which is the best fit to the real opacity of matter with solar abundances when hydrogen is fully ionized and bound-free absorption by heavy elements is present. Then the diffusion constant $D$ takes the form

$D=7.56 \times 10^{34} \alpha^{4 / 5}(\mu / 0.5)^{-3 / 4} \frac{M_{\mathrm{bh}}}{M_{\odot}}$.

The numerical constant in (3) incorporates the opacity coefficient $\kappa_{0}$ and a factor about 1 that accounts for the vertical structure solution (for more details see LS00; LSO2).

The key parameters for computing the emission of the disk are the effective temperature and the half-thickness of the disk, crucial for calculating self-irradiation. LS00 provides the halfthickness of the disk for the zones with Kramers opacity:

$$
\begin{aligned}
\frac{z_{0}(R)}{R}= & 0.135 \alpha^{-1 / 2}\left(\frac{M_{\mathrm{bh}}}{M_{\odot}}\right)^{-1 / 4}\left(\frac{R}{R_{\mathrm{out}}}\right)^{1 / 20} \\
& \times f(\xi)^{3 / 20}\left(\frac{R_{\mathrm{out}}}{R_{\odot}}\right)^{3 / 4}\left(\frac{t+\delta t}{10^{d}}\right)^{-1 / 2}
\end{aligned}
$$

Here the numerical constant contains a factor about 2.6 that accounts for the vertical structure solution and thus depends on the value $\kappa_{0}$ (see Suleimanov et al. 2007, and Eq. (36) therein). Function $f(\xi)$ is such that $y(\xi)=f^{\prime}(\xi)$ and

$f(\xi)=1.43 \xi-0.46 \xi^{7 / 2}+0.03 \xi^{6}$

Figure 1 shows the ratio $z_{0} / R$ versus radius obtained by solving the vertical structure equations with the tabulated opacity values calculated for the solar chemical abundance (Kurucz 1994; Suleimanov 1991; Lipunova \& Suleimanov 2004). It can be seen that the half-thickness of the disk calculated from the real (tabulated) opacity is reproduced satisfactorily by Eq. (4).

The effective temperature of the disk may be written as

$\sigma T_{\mathrm{vis}}^{4}(R, t)=\frac{3}{8 \pi} \dot{M}(0, t) \omega_{\mathrm{K}}^{2}(R) \Phi(R)$, 
where $\sigma$ is the Stephan-Boltzmann constant; $\omega_{\mathrm{K}}=\sqrt{G M / R^{3}}$; $\Phi(R)$ is a "structure function" such that for a stationary, nottruncated disk in the Newtonian limit $\Phi(R)=1-\sqrt{R_{\text {in }} / R}$. Taking general relativistic effects into account following Page \& Thorne (1974) and Riffert \& Herold (1995) with zero torque at the inner disk radius, we have

$$
\begin{aligned}
\Phi(R)= & {\left[\left(\frac{R}{R_{0}}\right)^{3} \frac{x-x_{0}-1.5 a \ln \frac{x}{x_{0}}-A-B-C}{x^{4}\left(x^{3}-3 x+2 a\right)}\right] } \\
& \times\left[\sqrt{\frac{R_{\text {out }}}{R}} \frac{f(\xi)}{y(0)}\right],
\end{aligned}
$$

where

$$
\begin{aligned}
& A=\frac{3\left(x_{1}-a\right)^{2}}{x_{1}\left(x_{1}-x_{2}\right)\left(x_{1}-x_{3}\right)} \ln \frac{x-x_{1}}{x_{0}-x_{1}}, \\
& B=\frac{3\left(x_{2}-a\right)^{2}}{x_{2}\left(x_{2}-x_{1}\right)\left(x_{2}-x_{3}\right)} \ln \frac{x-x_{2}}{x_{0}-x_{2}}, \\
& C=\frac{3\left(x_{3}-a\right)^{2}}{x_{3}\left(x_{3}-x_{1}\right)\left(x_{3}-x_{2}\right)} \ln \frac{x-x_{3}}{x_{0}-x_{3}}, \\
& x_{1}=2 \cos \left(\frac{\arccos a-\pi}{3}\right), \quad x_{2}=2 \cos \left(\frac{\arccos a+\pi}{3}\right), \\
& x_{3}=-2 \cos \left(\frac{\arccos a}{3}\right), \quad x_{0}=\sqrt{\frac{R_{\text {in }}}{R_{0}}}, \quad x=\sqrt{\frac{R}{R_{0}}},
\end{aligned}
$$

and $R_{0}=G M_{\mathrm{bh}} / c^{2}$. The term in the first square brackets of Eq. (7) represents a general relativistic correction and approaches 1 at large radii. The term in the second square brackets of Eq. (7) results from the non-stationary solution, and it equals approximately $1 / 1.43$ near the outer radius and $\approx 1$ at small radii.

At the outer edge of the disk there are additional sources of heating. Some type of collision between the disk and the incoming matter may cause a development of a hot area. Moreover, the tidal interactions rise steeply at $R_{\text {out }}$ (Ichikawa \& Osaki 1994) and lead to a corresponding energy release. We believe that neither of the above effects contributes significantly to the optical flux during the outburst and do not include them in the present study.

\subsection{X-ray spectrum}

In the vicinity of a compact relativistic object, the outgoing $\mathrm{X}$-ray spectrum is disturbed by the effects of Doppler boosting due to the disk rotation, gravitational focusing, and the gravitational redshift (Cunningham 1975). To calculate the spectrum of a disk, as detected by an observer at infinity, we use a computer code by Speith et al. (1995), which provides a transfer function allowing for these effects.

The transfer function is applied to the local X-ray spectra, which are diluted Planck spectra with a color temperature $T_{\mathrm{c}}(R)=f_{\mathrm{c}} T_{\text {vis }}(R)$ due to Compton scattering, where $f_{\mathrm{c}}=1.7 \pm$ 0.2 (Shimura \& Takahara 1995; Davis et al. 2005).

Figure 2 illustrates the general relativistic effects on the spectrum of the accretion disk around a Kerr black hole (the Kerr parameter $a=0.998)$. Shown are the spectrum as seen by a remote observer for a disk inclination $\theta=43^{\circ}(\cos \theta=0.73)$ and the spectrum for $\theta=87^{\circ}(\cos \theta=0.05)$. Naturally, the spectral shape of the X-ray emission received by the outer disk ( $\theta$ is large) differs significantly from what is seen by a remote observer. The intensity of the illuminating flux is significantly higher than calculated without general relativistic effects.
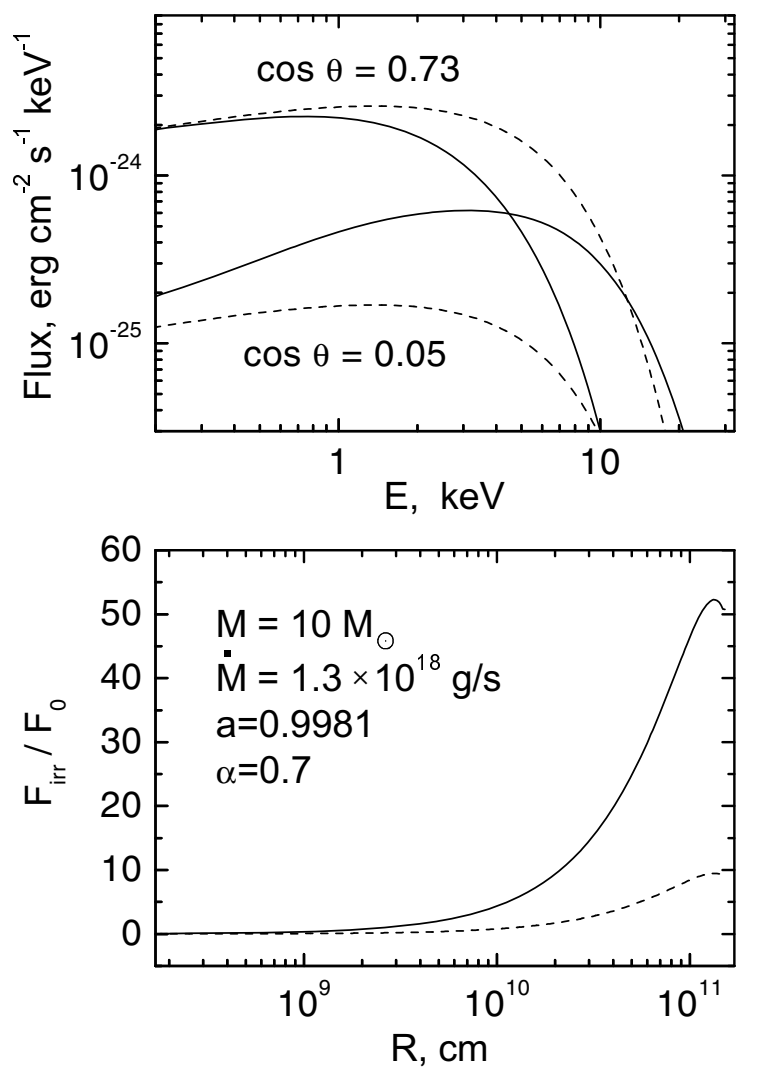

Fig. 2. Upper panel: X-ray spectra of the accretion disk around a Kerr black hole calculated for different disk inclinations. Lower panel: incident-to-intrinsic flux ratio vs. radius. For both panels the Kerr black hole has $a=0.998$. The solid lines show the impact of the relativistic effects on light propagation and can be compared with results obtained in the Newtonian limit shown by the dashed lines.

There are different methods of computing general relativistic effects on light propagation. An example of another implementation is one by Li et al. (2005), which is realized in the model "kerrbb" in the xspec package. However, kerrbb is restricted to inclination angles $\leq 85^{\circ}$. Thus we cannot readily use it to obtain the flux illuminating the outer disk, the calculation of which we describe in the following section.

To calculate the X-ray flux at the Earth we take interstellar absorption into account (Morrison \& McCammon 1983).

\subsection{Self-irradiation of the disk}

The flux falling onto a unit area that is normal to the emission outgoing at an angle $\theta$ to the disk axis, can be written as

$F(r, \theta)=\frac{L_{X}}{4 \pi r^{2}} \Psi(\theta)$

where $L_{\mathrm{X}}$ is the luminosity of the central X-ray source and $r$ the distance of the unit area from the center. In plain space-time geometry, for a flat disk as the central source, $\Psi(\theta)=2 \cos (\theta)$. We use the function $\Psi(\theta)$ provided by the code of Speith et al. (1995). Examples of $\Psi(\theta)$ for some values of the Kerr parameter are shown in Fig. 3

To calculate flux absorbed by a unit area of the disk, one should allow for not all of the incident flux being thermalized 


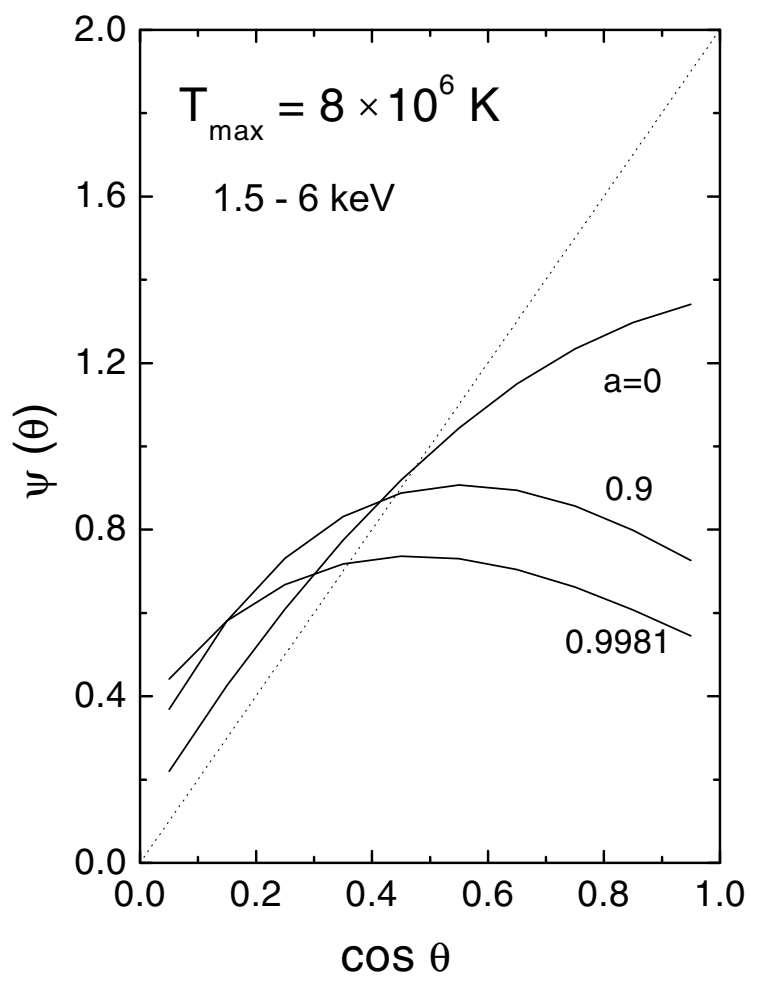

Fig. 3. Function $\Psi(\theta)$ for the different values of the Kerr parameter. See also Fig. 9 in Suleimanov et al. (2007).

(Shakura \& Sunyaev 1973; van Paradijs 1996; King \& Ritter 1998):

$F_{\mathrm{irr}}(R)=\eta \frac{L_{\mathrm{X}}}{4 \pi R^{2}} \Psi(\theta)\left[\frac{\mathrm{d} z_{0}}{\mathrm{~d} R}-\frac{z_{0}}{R}\right]$,

where $\eta$ is a thermalization coefficient, and the term in square brackets stands for the inclination of the disk surface to the incident rays. Assuming the disk to be geometrically thin, the distance $r$ is replaced by the radius of the disk $R$ at which the area in question is located.

The geometrical factor in the square brackets of Eq. (10) is remarkably different for the cases of the time-dependent model and a stationary disk. A stationary disk with $\dot{M}=\dot{M}(0, t)$ has approximately the same $z_{0} / R$ as in Eq. (4). However, value $\mathrm{d} z_{0} / \mathrm{d} R$ differs significantly. Using Eq. (4) we obtain the geometrical factor for a non-stationary, externally-truncated disk,

$\frac{\mathrm{d} z_{0}}{\mathrm{~d} R}-\frac{z_{0}}{R}=\frac{z_{0}}{R}\left(\frac{1}{20}+\frac{3}{40} \sqrt{\frac{R}{R_{\text {out }}}} \frac{y(\xi)}{f(\xi)}\right)$,

which is $\approx 1 / 20\left(z_{0} / R\right)$ for $R \approx R_{\text {out }}$, while the geometrical factor for a steady-state disk model (Shakura \& Sunyaev 1973) is $1 / 8\left(z_{0} / R\right)$; thus, the time-dependent disk near the outer radius is less irradiated.

The factor $\eta$ can be thought of as a coefficient for the reprocessing of incident X-rays ("reprocessing efficiency"), i.e. the fraction of X-rays absorbed in the under-photosphere layers. Values of $\eta$ suggested by researchers differ significantly. For low-mass X-ray binaries, de Jong et al. (1996) obtained a low value for reprocessing efficiency $\eta \sim 0.1$. In contrast, modeling the outbursts of GRS 1124-68 and A 0620-00, Esin et al. (2000) assume that almost all the incident $\mathrm{X}$-ray flux is thermalized, $\eta=0.8-0.9$.

Taking another approach, a value for the reprocessing efficiency can be derived from an accurate consideration of the atmosphere of an accretion disk exposed to the incident radiation (Suleimanov et al. 1999). For a disk with ionized hydrogen, a decisive factor is the spectrum of the incident X-rays. Only rather hard X-rays, of energy $E>2 \mathrm{keV}$, penetrate the layers where the optical continuum is produced. Softer X-rays are absorbed higher in the atmosphere and reprocessed to the far UV (Suleimanov et al. 1999; El-Khoury \& Wickramasinghe 1999). To raise the optical emission from the disk, the temperature should increase at the depths where the optical continuum is formed. However, most of the X-rays with energies over $10 \mathrm{keV}$ are reflected due to electron scattering. Thus, it is mainly X-rays in the interval 2-10 keV that are reprocessed to optical emission. The high/soft spectral state of X-ray novae is characterized by soft X-ray spectra with a corresponding black body temperature $~ 0.5-1.0 \mathrm{keV}$. Using Eq. (22) from Suleimanov et al. (1999) we obtain low values of the reprocessing efficiency, 0.1-0.2.

Esin et al. (2000) generally conclude that the outer parts of accretion disks in the high/soft state should be significantly thicker than follows from the standard model of Shakura \& Sunyaev (1973). Concerning this result we bring up the following argument. The irradiation cannot increase the thickness of the disk beyond the values predicted by Eq. (4), either in the regions where Kramers opacity applies, or in the regions with partly ionized hydrogen (Dubus et al. 1999; Suleimanov et al. 2007). However, a layer of hot coronal gas could be present above the disk due to the irradiation of the outer disk by hard X-rays (Begelman et al. 1983; Begelman \& McKee 1983; Ko \& Kallman 1994; Różańska \& Czerny 1996; Proga \& Kallman 2002; Jimenez-Garate et al. 2002). Such a corona, optically thick in the radial direction, effectively increases the geometrical thickness of the disk and augments the degree of self-irradiation. Furthermore, the corona could be inhomogeneuos and contain optically thick gas blobs as proposed for supersoft X-ray sources by Suleimanov et al. (2003). In this case the reprocessing efficiency can increase significantly due to multiple scattering between the blobs.

There is another possibility for increasing a disk's thickness. Recent simulations of magnetorotational turbulence (e.g. Miller \& Stone 2000; Turner 2004; Hirose et al. 2006) show that magnetic pressure is significant in the upper layers of the disk, probably resulting in a vertically extended atmosphere. Thus, the optical-depth unity surface can be located a factor of two above the "standard" disk surface (Hirose et al. 2006).

In view of the present uncertainty, we consider the reprocessing efficiency as a parameter. For the same reason, we do not calculate the half-thickness of the disk using the tabulated values of opacity coefficient but utilize Kramers approximation. In Eq. (10), the presence of the corona is treated as the increased thickness of the disk, which equals $z_{0}$ multiplied by a chosen factor.

It should be noted that consideration of the hard spectral component, which is emitted by a central source (possible central corona) and characterized by $\Psi(\theta) \approx 1$, would enhance the self-irradiation of the disk.

\subsection{Optical emission from the disk}

The spectrum of the time-dependent disk at any moment of time is calculated by integrating local spectra over radius:

$L_{\lambda}=4 \pi^{2} \int_{R_{\text {in }}}^{R_{\text {out }}} B_{\lambda}(T) R \mathrm{~d} R$ 


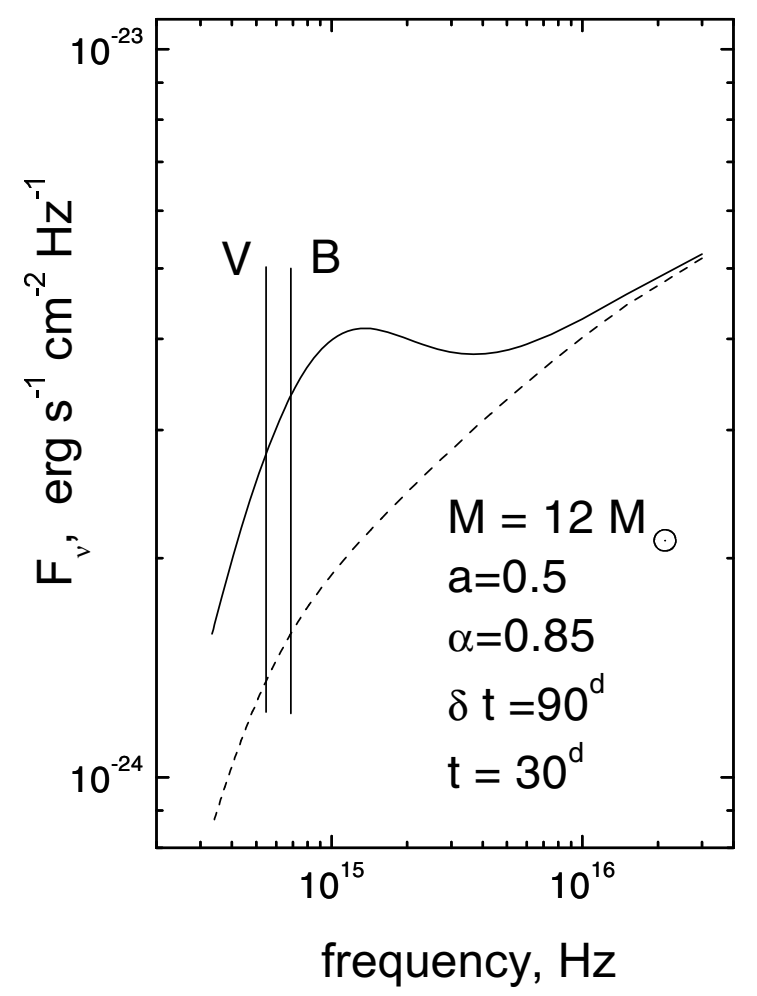

Fig. 4. Optical and UV spectrum of a modeled disk of A 0620-00 with self-irradiation (the solid line) and without (the dashed line). Vertical lines denote the positions of the centers of $B$ and $V$ bands.

At the outer parts of the disk the local spectra are Planck functions with an effective temperature $T$ increased due to the intercepted X-ray flux

$\sigma T^{4}=\sigma T_{\text {vis }}^{4}+F_{\text {irr }}$,

where $T_{\text {vis }}$ is the effective temperature (Eq. (6)) in the absence of X-ray irradiation. The spectral flux density at the Earth is given by

$f_{\lambda}=\frac{L_{\lambda}}{2 \pi d^{2}} \cos i \exp \left(-\tau_{\lambda}\right)$,

where $d$ the distance to the system, $i$ the inclination of the disk, $\tau_{\lambda}$ is the optical depth for interstellar absorption.

The unabsorbed optical and UV spectrum of a self-irradiated disk is shown in Fig. 4.

\section{Modeling procedure}

The physical parameters of X-ray novae (mass, Kerr parameter, viscosity parameter $\alpha$, etc.) are sought by comparing modeled and observed light curves. A particular model of the disk is defined by a set of input physical parameters. Some of these can be varied and some are assumed to be known from observations.

To compare the model with observations, we chose several values calculated in a specific way from observational data "fitted light curve parameters" - and fit them by the model.

\subsection{Fitted light-curve parameters}

As shown in LS02, the X-ray and optical light curves of the $\mathrm{X}$-ray novae considered are represented well by linear functions in "log(flux)-time" coordinates throughout the first 40-60 days after the peak. Thus each light curve can be described by a pair of parameters: the slope and the flux at a reference time, which are to be fitted. This approach allows one to significantly reduce the computer time required for finding satisfactory model parameters.

In the course of the modeling, we reproduce the following values: the slope of the X-ray light curve $a_{\mathrm{X}}$, the slope of the optical light curve in the $B$ band $a_{B}$, the X-ray flux $F_{\mathrm{X}}$, the flux $F_{B}$, the color index $B-V$, and, optionally, $U-B$. All parameters, except for the first two, are calculated for a reference time.

\subsection{Input parameters of the model}

The free input parameters are: the black hole mass $M_{\mathrm{bh}}$, its Kerr parameter $a$, the viscosity parameter $\alpha$ of the disk, the time parameter $\delta t$ to fix the accretion rate at time $t=0$, and the reprocessing efficiency $\eta$. Other variable parameters of the model are the color-to-effective temperature ratio $f_{\mathrm{c}}$ and the ratio $z / z_{0}$, where $z$ is the height of X-rays' interception.

The parameters that are more or less fixed include: the optical component mass $M_{\mathrm{o}}$, the mass function $f(M)$, the period of the binary $P$, and the interstellar absorption, expressed by parameter $N_{\mathrm{H}}$ for an X-ray band (the number of $\mathrm{H}$ atoms along the line of sight per $\left.\mathrm{cm}^{2}\right)$ or by the color excess $E(B-V)$ for optical bands.

The mass of the optical component $M_{\mathrm{o}}$ and the interstellar absorption are not very precisely known. In LS02 they were varied within narrow limits, and it was shown that the effect of the variation was small, so we fix their values.

Given the binary mass, one obtains the semi major axis of the binary system

$\tilde{a}=\left(\frac{G\left(M_{\mathrm{bh}}+M_{\mathrm{o}}\right) P^{2}}{4 \pi^{2}}\right)^{1 / 3}$

and can calculate the outer radius of the disk approximately. We take this equal to $80 \%$ of the radius of a sphere whose volume equals that of the black hole's Roche lobe (Eggleton 1983):

$R_{\mathrm{out}}=0.8 \tilde{a} \frac{0.49 q^{2 / 3}}{0.6 q^{2 / 3}+\ln \left(1+q^{1 / 3}\right)}$,

where $q=M_{\mathrm{bh}} / M_{\mathrm{o}}$. This approximation agrees with calculations by Paczynski (1977) and with the direct estimates of disk sizes in cataclysmic variables (Sulkanen et al. 1981). One can also derive the inclination $i$ of the binary plane:

$i=\arcsin \left(\frac{f(M)\left(M_{\mathrm{bh}}+M_{\mathrm{o}}\right)^{2}}{M_{\mathrm{bh}}^{3}}\right)^{1 / 3}$.

It is necessary to mention that the inner disk can have another inclination to the line of sight. The black hole angular momentum can be misaligned with that of a binary due to asymmetric previous supernova explosion. In this case the inner disk will tilt toward to equatorial plane of the black hole (Bardeen \& Petterson 1975). We do not consider this possibility in the present study and assume that the inner and the outer parts of the disk have the same inclination; therefore, some of our results can be biased, black hole angular momentum in particular.

The distance to the system is defined in the course of the modeling. As the limits on black hole masses and distances are known from observations, they are used to restrict the allowed ranges of other parameters. 


\subsection{Qualitative investigation of nonstationary accretion in $\alpha$-disks}

Before proceeding to the model results, we discuss the qualitative features of the nonstationary model.

An estimate of $R_{\text {out }}$ can be derived from Eqs. (15) and (16) in the approximation that $M_{\mathrm{bh}} \gg M_{\mathrm{o}}$

$R_{\text {out }} \propto M_{\mathrm{bh}}^{1 / 3} P^{2 / 3}$.

Using this relation and Eq. (1), we obtain

$\dot{M} \propto \frac{M_{\mathrm{bh}}^{20 / 9} P^{25 / 9}}{\alpha^{8 / 3}(t+\delta t)^{10 / 3}}$.

Next, the bolometric luminosity of the disk can be written as

$L_{\mathrm{bol}}=\varepsilon(a) \dot{M} c^{2} \approx 2 \pi \sigma\left(\frac{T_{\mathrm{X}}}{f_{\mathrm{c}}}\right)^{4} R_{\mathrm{X}}^{2}$,

where $\varepsilon(a)$ is the efficiency of conversion of gravitational energy to radiation, dependent on the dimensionless Kerr parameter $a$; $T_{\mathrm{X}}$ is the maximum color temperature at radius

$R_{\mathrm{X}} \sim R_{\mathrm{in}} \propto \beta(a) M_{\mathrm{bh}}$.

Here $\beta(a)$ represents a dependence on the Kerr parameter. For a Schwarzschild black hole $a=0, \epsilon=0.057, \beta=6$ and for a maximally rotating Kerr black hole $a=1, \epsilon=0.42, \beta=$ 1. Recall that the color temperature is $f_{\mathrm{c}}$ times greater than the effective temperature. From Eqs. (19) and (20) we have

$T_{\mathrm{X}} \propto \frac{\varepsilon^{1 / 4}}{\beta^{1 / 2}} \frac{M_{\mathrm{bh}}^{1 / 18} P^{25 / 36}}{\alpha^{2 / 3}(t+\delta t)^{5 / 6}}$.

Ebisawa et al. (1994) obtained the maximum color temperature variations for GRS 1124-68. Figure 5 shows good agreement between the observed decrease and the theoretical dependence on time (Eq. (22)).

The fading of an X-ray light curve is faster than that of a bolometric light curve, expressed by Eq. (19). An interval of the spectrum that we consider can be approximated by Wien's law with a temperature close to the maximum color temperature of the disk. Thus, variation in the X-ray flux of a soft X-ray transient is governed by the decreasing color temperature and the associated shift of the Wien-like spectrum to lower energies; a well-known exponential dependence of the light curve on time emerges (LSO0). The optical light curves have flatter slopes because the main decrease in the disk luminosity happens at high energies.

Let us calculate the slope of an optical light curve, for example, in the $B$ band. Because the optical flux from the disk is mainly determined by the reprocessed X-ray emission from the central source, we can write

$L_{B} \propto R_{\text {out }}^{2} T_{\text {irr }}^{k}$,

where index $k \geqslant 1$ comes from a relation between the optical flux and the effective temperature. For instance, in the Rayleigh-Jeans part of the spectrum $k=1$. The time dependence of the effective temperature at the outer edge of the disk can be obtained from Eq. (10):

$\sigma T_{\text {irr }}^{4} \propto \eta \frac{L_{\text {bol }}}{R_{\text {out }}^{2}}\left(\frac{z_{0}}{R_{\text {out }}}\right)^{2}$

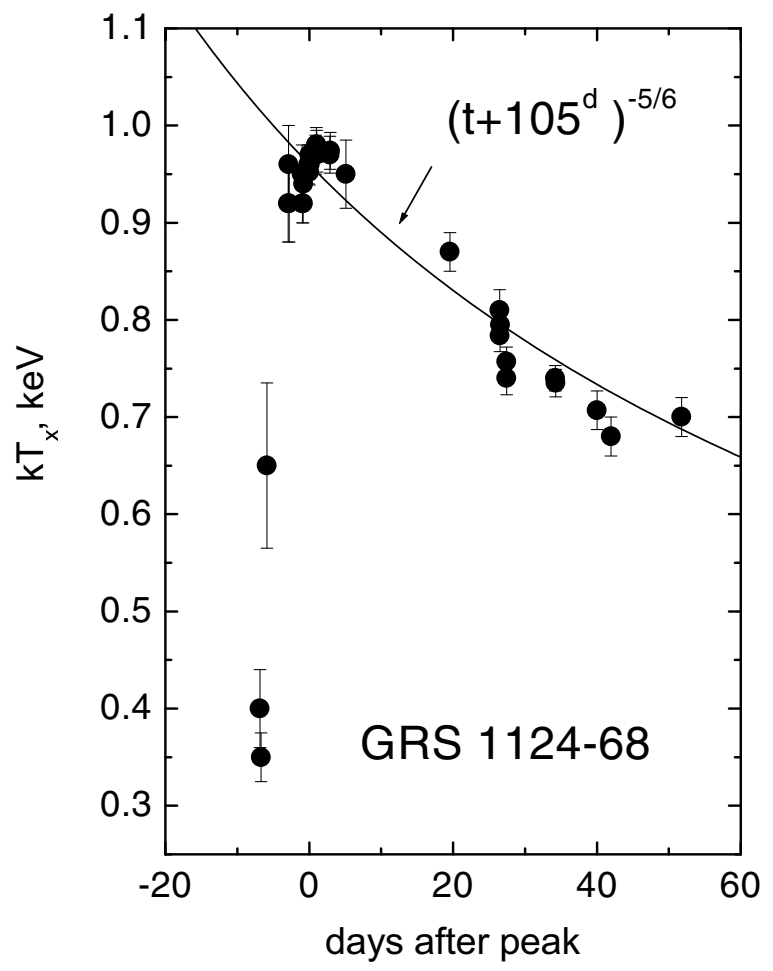

Fig. 5. Observed maximum color temperature of the disk in GRS 1124-68 (Ebisawa et al. 1994) vs. time in comparison with Eq. (22) where $\delta t=105$ days expressed in seconds.

or, using Eqs. (4), (19), and (20),

$T_{\text {irr }} \propto(\varepsilon \eta)^{1 / 4} \frac{M_{\mathrm{bh}}^{7 / 18} P^{11 / 18}}{\alpha^{11 / 12}(t+\delta t)^{13 / 12}}$.

The slope of the $B$ light curve at time $t$ is

$a_{B}=\frac{\mathrm{d} \lg L_{B}(t)}{\mathrm{d} t}=-\frac{13}{12} \frac{k \lg \mathrm{e}}{t+\delta t}$,

and is essentially determined only by the parameter $\delta t$ and vice versa. Our numerical estimations yield $k \approx 1.9$ for the spectrum of an irradiated disk. Substituting values of slopes $a_{B}$ from Table 1 into Eq. (26), we obtain that $\delta t$ is approximately 90 and 100-110 days for A 0620-00 and GRS 1124-68 (for the 30th and the 50th days after the peak, respectively).

The color index $B-V$ obviously determines the temperature at the outer disk edge $T_{\text {irr }}\left(R=R_{\text {out }}\right)$. Thus, the parameters $a, M_{\mathrm{bh}}$, and $\alpha$, which produce a satisfactory model, should be related to meet the condition $T_{\text {irr }}\left(R=R_{\text {out }}\right) \approx$ const., defined by Eq. $(25)$. To conclude, we note a basic feature: for a constant black hole mass and $T_{\mathrm{X}}$, an increase in the Kerr parameter will cause the distance to the binary to decrease (cf. Eqs. (20) and (21)).

\section{Outbursts of Nova Mon 1975 (A 0620-00) and Nova Mus 1991 (GRS 1124-68)}

Observational parameters for Nova Mon 1975 and Nova Mus 1991 are summarized in Table 1 . We take $B$ and $V$ optical light curves collected in LS02. The $U$ light curve of A 0620-00 is taken from Liutyi (1976), van den Bergh (1976), and Duerbeck \& Walter (1976) and transferred to units of $\mathrm{erg} \mathrm{cm}^{-2} \mathrm{~s}^{-1}$ in the same way as described in LS02.

By and large, the outbursts are very much alike. Let us point out the distinctions. In GRS 1124-68, all light curves decline 
Table 1. Observed input parameters and fitted light curve parameters ${ }^{a}$.

\begin{tabular}{lll}
\hline \hline Parameter & A 0620-00 & GRS 1124-68 \\
\hline$P$ [days] & $0.322^{1,2}$ & $0.433^{5}$ \\
$f(M)$ & $2.7 M_{\odot}^{1,2}$ & $3.0 M_{\odot}^{5}$ \\
$M_{\mathrm{o}}$ & $0.5 M_{\odot}$ & $0.8 M_{\odot}$ \\
$N_{\mathrm{H}}[$ atoms cm \\
$E(B-V)$ & $3 \times 10^{21 \mathrm{~b}, \mathrm{c}}$ & $1.4 \times 10^{21 \mathrm{c}, 6}$ \\
$a_{\mathrm{X}}$ & $0.35^{3}$ & $0.3^{\mathrm{c}}$ \\
$F_{\mathrm{X}}$ & $-0.0149 \pm 0.0004$ & $-0.0125 \pm 0.0005$ \\
$a_{B}$ & 100 photons s$^{-1} \mathrm{~cm}^{-2}$ & $6.8 \times 10^{-8} \mathrm{erg} \mathrm{s}^{-1} \mathrm{~cm}^{-2}$ \\
$F_{B}$ [erg s cm \\
$B-V$ & $-0.0074 \pm 0.0003$ & $-0.0060 \pm 0.0004$ \\
$B-V$ & $(1.24 \pm 0.07) \times 10^{-10}$ & $(1.12 \pm 0.03) \times 10^{-11}$ \\
$U-B$ & $0.24 \pm 0.03^{4}$ & $0.27 \pm 0.07^{4}$ \\
\hline
\end{tabular}

${ }^{a}$ Notations are explained in Sects. 3.1 and 3.2. Absolute fluxes are derived for the 30th day after the outburst peak. The slopes of the X-ray light curves and the X-ray fluxes correspond to different X-ray intervals: $1.5-6 \mathrm{keV}$ for A $0620-00$ and $1.2-6 \mathrm{keV}$ for GRS 1124-68.

${ }^{b}$ Intermediate between the values obtained by Weaver \& Williams (1974) and Wu et al. (1983).

${ }^{c} N_{\mathrm{H}}$ is obtained from $E(B-V)$ following Zombeck (1990).

References. (1) Cherepashchuk (2000); (2) Chen et al. (1997); (3) Wu et al. (1983); (4) Lipunova \& Shakura (2002); (5) Orosz et al. (1996); (6) della Valle et al. (1991); Cheng et al. (1992); Shrader \& Gonzalez-Riestra (1993).

more slowly: the decay time scales, or " $e$-folding times", of the X-ray and optical light curves are 35 days and 68 days for GRS $1124-68$ as against 30 days and 57 days for A 0620-00. In addition, the optical-to-X-ray flux ratio at the peak in A 0620-00 is $\sim 2$ times higher than in GRS 1124-68. The color index $(B-V)$ is greater for GRS 1124-68 (the object is "redder"), although the color excess $E(B-V)$ is higher for A 0620-00.

The input parameters of the model are varied within the following limits: $M_{\mathrm{bh}}: 5-15 M_{\odot}$, the Kerr parameter $a$ : $0-0.9981$, the $\alpha$-parameter: $0.1-1$, the time normalization parameter $\delta t$ : 80-140 days. The modeling results critically depend on the reprocessing efficiency $\eta$, which we vary between 0 and 1 .

\subsection{A 0620-00. Importance of irradiation}

We use the 1.5-6 keV light curve obtained with the SAS-3 CSL-A (Kuulkers 1998). The flux data are converted from counts $\mathrm{s}^{-1}$ to photons $\mathrm{cm}^{-2} \mathrm{~s}^{-1}$ assuming a Crab-like spectrum. The following normalizations are adopted: $1 \mathrm{Crab} \approx$ 136 counts/s (Buff et al. 1977), $1 \mathrm{Crab} \approx 5$ photons $\mathrm{cm}^{-2} \mathrm{~s}^{-1}$ in $1.5-6 \mathrm{keV}$. We believe the resulting error to be acceptable because 1.5-6 keV and 3-6 keV fluxes in Crab units are close to each other (Kaluzienski et al. 1977), and thus the spectral shapes of A 0620-00 and the Crab should be similar in the X-ray range in question. In Fig. 6 we compare the X-ray spectrum calculated from the model with the spectrum observed by Long \& Kestenbaum (1978). The latter was obtained on the 70th day after the peak, when the flux had the same magnitude as on the 50th day (Kuulkers 1998). We conclude that the chosen normalizations are satisfactory.

During the interval of 20-40 days after the peak the multitemperature black body disk component dominates the spectrum (see LS02). In Table 1 the slopes of the light curves are calculated for this time interval, and values $F_{\mathrm{X}}$ and $F_{B}$ correspond to the 30th day.

In LS02 the difficulty of reproducing the slope of the optical light curves of A 0620-00 was revealed. Including reprocessed emission helps to solve the issue. In the case of the self-irradiated

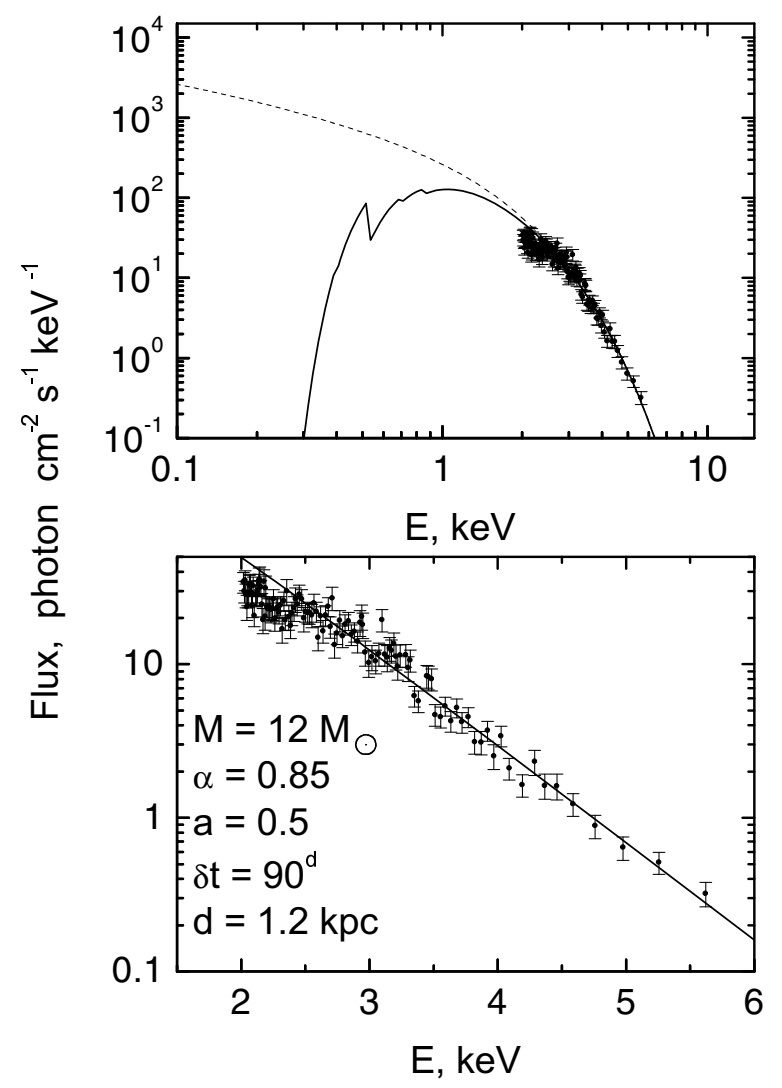

Fig. 6. Comparison of the observed spectrum of A 0620-00 (Long \& Kestenbaum 1978) and the modeled one for $t=50^{\mathrm{d}}$. The solid line represents the case when interstellar absorption is taken into account with $N_{\mathrm{H}}=3 \times 10^{22}$ atoms $\mathrm{cm}^{-1}$; the dashed line shows the unabsorbed modeled spectrum. The lower panel shows a enlarged area from the upper panel.

disk, the slope of the optical light curve steepens, partly because the incident flux declines faster with time than the intrinsic flux, but mainly because $\delta t$ has to be decreased to adjust the optical-to-X-ray flux ratio, and $\delta t$ is uniquely related to the slope (see Eq. (26)). Modeling results are presented in Fig. 7. The relation between allowable values of the black hole mass, the Kerr parameter, and the $\alpha$-parameter reflects the constancy of the value $T_{\mathrm{X}}$. An example of the modeled and observed light curves is shown in Fig. 8.

There are two parameters that define the effect of irradiation (see Eq. (10)): the reprocessing efficiency $\eta$ and the ratio $z / z_{0}$. In the Newtonian limit the combination $\eta\left(z / z_{0}\right)^{2}$ is decisive, but in general relativity this is correct only approximately due to the relativistic function $\Psi(\theta)$. Our calculations show that the observed parameters can be fitted with $\eta\left(z / z_{0}\right)^{2} \sim 1-2$. Evidently, the reprocessing efficiency $\eta$ can hardly be as high as 1 . Therefore, the accretion disk in this X-ray nova must have an effective geometrical thickness greater than that given by Eq. (4). One can introduce the so-called irradiation parameter $C$ to rewrite Eq. (10): $F_{\text {irr }}(R)=C\left(L_{\mathrm{X}} / 4 \pi R^{2}\right)$ (see, for example, Dubus et al. 2001). For the model presented in Fig. 8, in the time span 10-50 days, the irradiation parameter, calculated at the outer radius $R_{\text {out }}=1.59 \times 10^{11} \mathrm{~cm}$, changes in the range $\sim(8-6) \times 10^{-4}$.

There are no satisfactory models with the Kerr parameter $a>$ 0.7 for $f_{\mathrm{c}}=1.7$ (Fig. 7). We also investigate the influence of parameter $f_{\mathrm{c}}$. Results with $f_{\mathrm{c}}=1$ are presented in Fig. 7 by diamonds (for $\eta=1$ and $\left.z / z_{0}=1\right)$ and triangles $(\eta=0.5$ and 


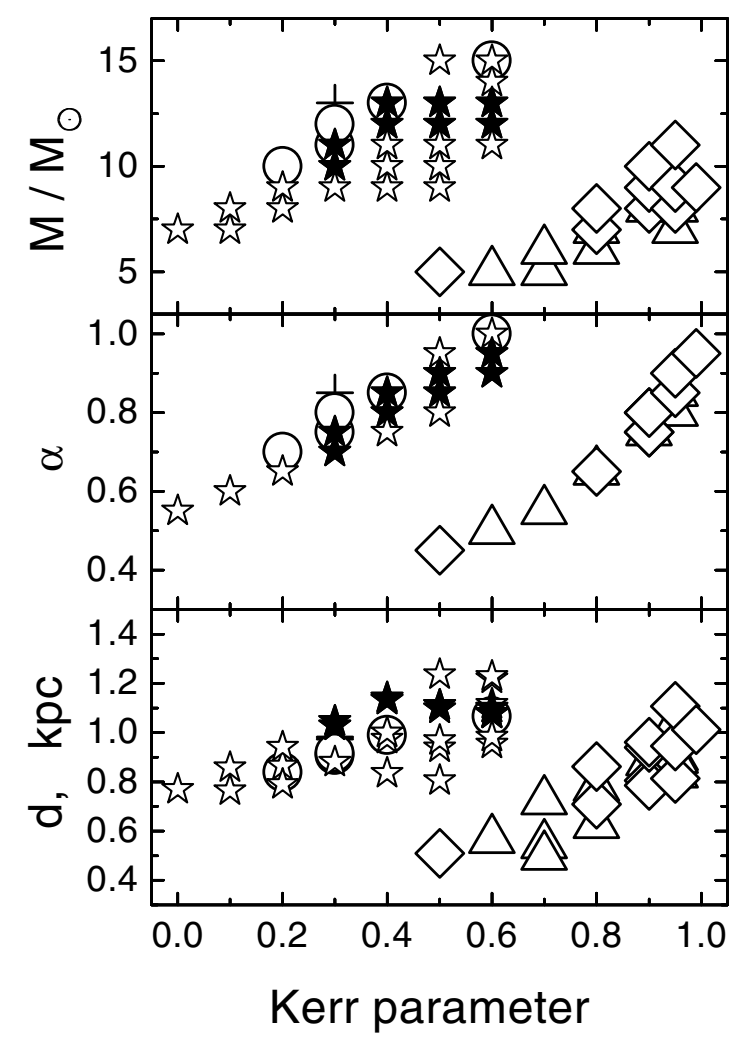

Fig. 7. Resulting parameters of A $0620-00$ vs. Kerr parameter. Models with $f_{\mathrm{c}}=1.7$ are denoted by circles $\left(z=z_{0}\right.$ and $\left.\eta=1\right)$, stars $\left(z=2 z_{0}\right.$ and $\eta=0.5)$, and pluses $\left(z=2 z_{0}\right.$ and $\eta=0.5$ with limb darkening). Models with $f_{\mathrm{c}}=1$ are denoted by diamonds $\left(z=z_{0}\right.$ and $\left.\eta=1\right)$ and triangles $\left(z=2 z_{0}\right.$ and $\left.\eta=0.5\right)$. Models denoted by filled stars have masses and distances in agreement with Gelino et al. (2001a).

$z / z_{0}=2$ ). One can see that models with $f_{\mathrm{c}}=1$ have higher values of the Kerr parameter, which compensate the decrease in the color temperature. Other parameters (the black hole masses, $\alpha$-parameter, and the distance), though somewhat decreased, are still in the same range. Therefore, any uncertainty in $f_{\mathrm{c}}$ leads mainly to a deviation of the Kerr parameter.

In some of the models, the effect of limb darkening was included. For the local X-ray spectra, the limb darkening was taken into account assuming the dominant electron scattering, and the grey atmosphere (Eddington) approach was used for the local optical spectra (Chandrasekhar 1950; Sobolev 1949, 1969). The corresponding results are shown in Fig. 7 by pluses. Taking account of limb darkening leads to some increase in the black hole mass, the $\alpha$-parameter, and the distance to the binary, not altering the results qualitatively.

Independent estimates of the system parameters can be used to constrain our results further. Available in the literature are estimates of the distance $d=1 \pm 0.4 \mathrm{kpc}$ (Shahbaz et al. 1994; Barret et al. 1996) and the black hole mass: $M_{\mathrm{bh}}=10 \pm 7 M_{\odot}$ (Shahbaz et al. 1994), $M_{\mathrm{bh}}=4.6 \pm 1.1 M_{\odot}$ (Haswell et al. 1993). The highest quoted accuracy is of the estimates given by Gelino et al. (2001a): $M_{\mathrm{bh}}=11 \pm 1.9 M_{\odot}$ and $d=1.116 \pm 0.114 \mathrm{kpc}$. The constructed models agree with these values if $\alpha=0.7-0.95$ and the Kerr parameter $a=0.3-0.6$ (Fig. 7).

\subsection{GRS $1124-68$}

Ebisawa et al. (1994) fit the spectra obtained during the 1991 outburst of GRS 1124-68 with a model comprising two

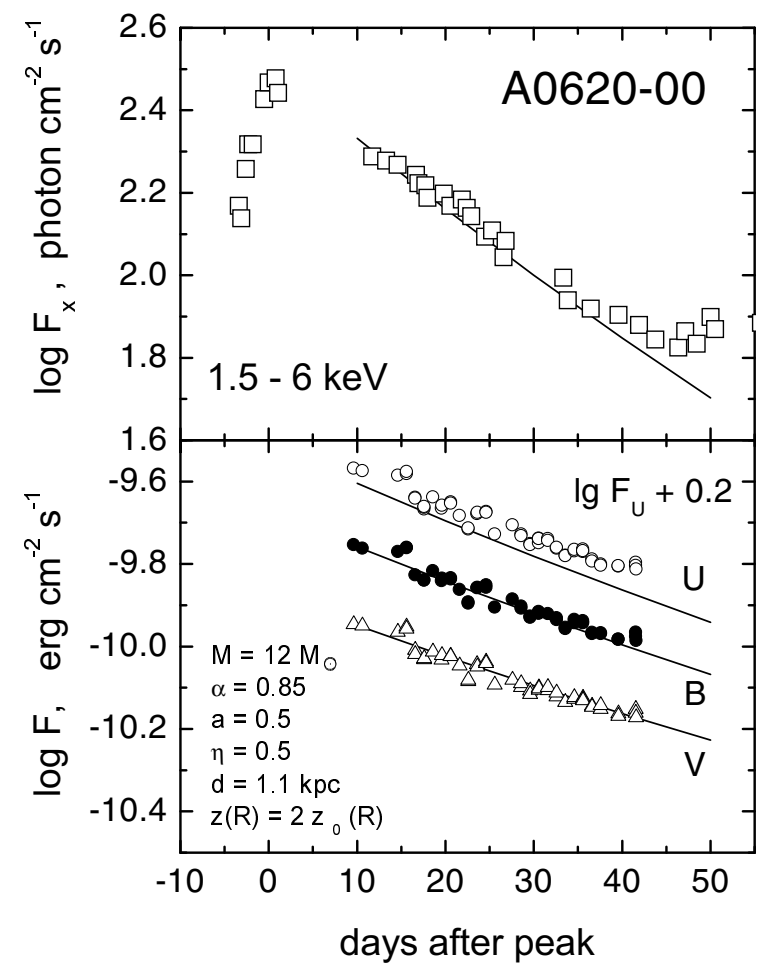

Fig. 8. Comparison of the modeled and observed light curves of A 0620-00. The $U$ flux is shifted upward by 0.2 .

components: a hard power-law component and a soft component emitted by a multicolor disk (Mitsuda et al. 1984). They present a time evolution of model parameters: the maximum temperature and the inner radius of the multicolor disk, as well as the photon index and the hard $2-20 \mathrm{keV}$ flux from the power-law component. This information allows us to calculate the unabsorbed 1.2-6 keV flux. In Table 1 the slopes of the light curves are calculated for a $20-50$ day interval.

It is possible to fit the light curves with plausible values of reprocessing efficiency, $\eta \lesssim 0.5$, keeping the standard thickness of the disk. For this system the relation $\eta\left(z / z_{0}\right)^{2} \sim 0.5-0.6$ is fulfilled. One gets almost same results with three different sets of parameters: (1) $z=z_{0}, \eta=0.5$; (2) $z=1.5 z_{0}, \eta=0.25$; (3) $z=2 z_{0}, \eta=0.15$ (see Figs. 9 and 10). The parameter $\delta t$ lies in the range from 100 to 115 days in all models. Figure 9 presents allowable intervals for the mass, the Kerr parameter, the $\alpha$-parameter, and the distance, which lies in the interval from 2.5 to $6 \mathrm{kpc}$.

Figure 11 illustrates the modeled light curves for parameters $M_{\mathrm{bh}}=8 M_{\odot}, \alpha=0.55, a=0, \delta t=115^{\mathrm{d}}, \eta=0.25$, and $z=1.5 z_{0}$. During the interval of 10-60 days, the irradiation parameter $C$ (see Sect. 4.1), calculated at the outer radius $R_{\text {out }}=$ $1.62 \times 10^{11} \mathrm{~cm}$, decreases from $\sim 3 \times 10^{-4}$ to $\sim 2.5 \times 10^{-4}$.

\subsubsection{Distance to GRS 1124-68}

Independent estimates of the distance and the black hole mass are: $d=3.4 \pm 0.6 \mathrm{kpc}$ and $M_{\mathrm{bh}}=7.5 \pm 3.5 M_{\odot}$ (Shahbaz et al. 1997), $d=5.5 \pm 1 \mathrm{kpc}$ and $M_{\mathrm{bh}}=5-7.5 M_{\odot}$ (Orosz et al. 1996). The highest accuracy for the black hole mass is claimed by Gelino et al. (2001b) and Khruzina et al. (2003): $M_{\mathrm{bh}}=6.95 \pm 0.6 M_{\odot}$ and $M_{\mathrm{bh}}=7.15 \pm 0.45 M_{\odot}$, respectively. Gelino et al. (2001b) estimate the distance as $5.1 \mathrm{kpc}$ or even 5.9 kpc (Gelino 2004). 


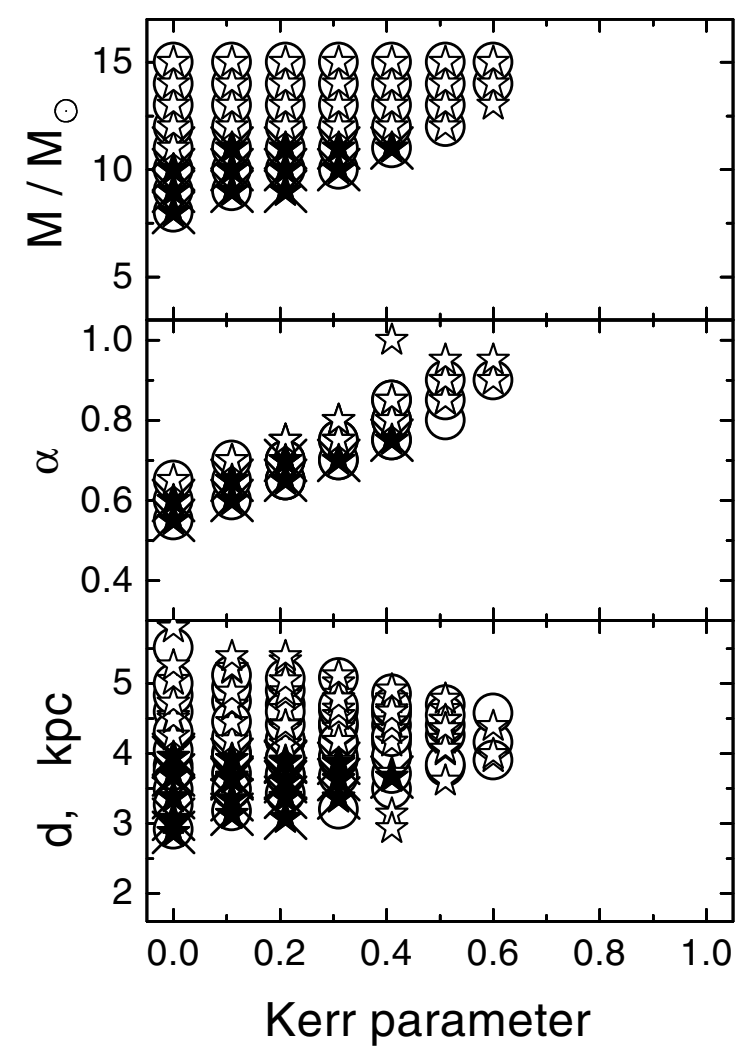

Fig. 9. Resulting parameters of GRS 1124-68 vs. Kerr parameter. Circles denote the models with $\eta=0.5, z=z_{0}$; stars: $\eta=0.25$, $z=1.5 z_{0}$; filled stars agree with the limits on the black hole mass (see explanation in Sect. 4.2.1); crosses: models with $\eta=0.15, z=2.0 z_{0}$ and masses within the same limits. All models have $f_{\mathrm{c}}=1.7$.

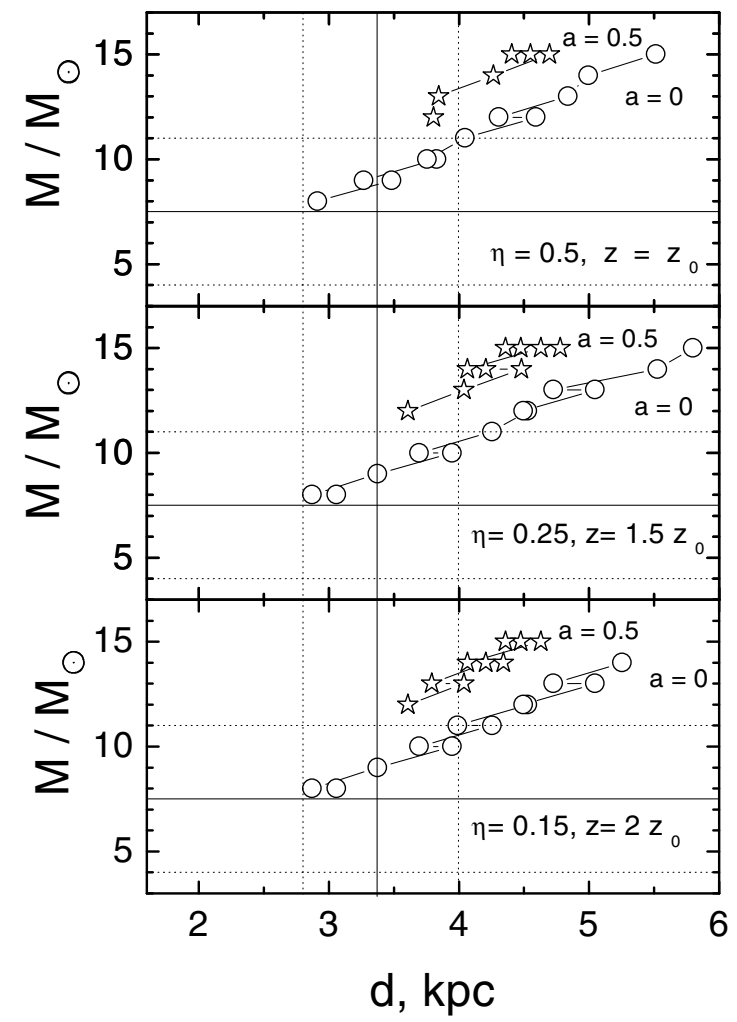

Fig. 10. Resulting relationships between the mass of the black hole in GRS 1124-68 and the distance to the system, for different values of $\eta$, $z / z_{0}$, and the Kerr parameter. Horizontal and vertical lines show observational limits and central values.

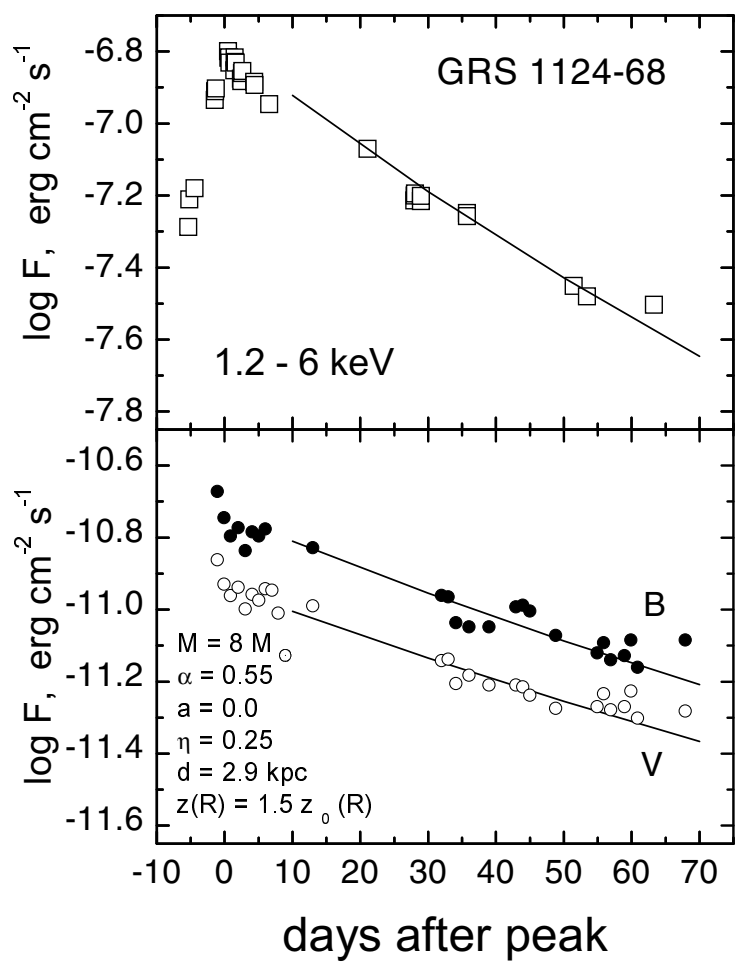

Fig. 11. Comparison of the modeled and observed light curves of GRS 1124-68.

The constructed models cannot agree with the distances obtained by Gelino et al. (2001b) and Gelino (2004), tending more to the estimates of Shahbaz et al. (1997). For the black hole mass $M=7.5 \pm 3.5 M_{\odot}, z=(1.5-2) z_{0}$, and $\eta=0.15-0.25$, we come to the following allowable intervals of parameters: the distance $3-4 \mathrm{kpc}$, the Kerr parameter $a \lesssim 0.4$, and the $\alpha$-parameter $0.55-0.75$ (Fig. 9).

The above estimates of the distance are derived from an analysis of light curves and spectra in optical and IR bands obtained during the quiescent state of the X-ray nova. The basic element for determining the distance in quiescence is the spectral class of the secondary. It is found from optical spectra ( $\sim \mathrm{K} 4 \mathrm{~V}$, Orosz et al. 1996) and yields a corresponding effective temperature ( $4500 \mathrm{~K})$. However, the optical light curves of the secondary imply a reflection, and it is necessary to assume $L_{\mathrm{irr}} \sim L_{\mathrm{opt}}$ (Khruzina et al. 2003). Thus, the apparent spectral class of the secondary is biased; if the actual effective temperature of the secondary is lower, then the binary is closer.

Another way to constrain the distance is to study X-ray spectra obtained during the outburst. Ebisawa et al. (1994) obtain a non-relativistic relation between the black hole mass and the distance, which can be rewritten as follows:

$d=2.6\left(\frac{f_{\mathrm{c}}}{1.7}\right)^{-2}\left(\frac{R_{\mathrm{in}}}{6 R_{0}}\right)\left(\frac{M_{\mathrm{bh}}}{10 M_{\odot}}\right)\left(\frac{\cos i}{0.5}\right)^{1 / 2} \mathrm{kpc}$.

This relation appears to agree with our results (Fig. 9), keeping in mind that slight changes due to relativistic corrections might be present.

It is worth noting here the possibility of increasing a distance obtained from X-ray data. The inner disk radius can be greater than the radius of the last marginally stable orbit $R_{\mathrm{ms}}$. This phenomenon is discussed for other Galactic black hole candidates (Gilfanov et al. 1999; Chaty et al. 2003). The inner region, evacuated by the standard disk, becomes hot and optically thin, so not 
contributing to the soft X-ray flux. We tested a number of models with the inner radius moved away up to $10 R_{\mathrm{ms}}$. The imposed boundary condition was that the viscous stress tensor becomes zero at $R_{\mathrm{ms}}$ and is non-zero at the inner radius $R_{\text {in }}$. We came to the conclusion that all such models, which satisfactorily fitted the data, had higher values of the Kerr parameter and unchanged values of the mass and the distance.

\section{Discussion and conclusions}

As shown, the model with non-stationary accretion onto a compact binary component, developed for an $\alpha$-disk in LS00, can account for the X-ray and optical light curves of the two X-ray novae considered, A 0620-00 and GRS 1124-68, in the high/soft state. Dedicated modeling of the light curves has previously been attempted in LS02. In the present study we extend the modeling by including the effects of general relativity on light propagation near black holes and self-irradiation of the disks.

One needs to assume a rather large contribution of reprocessed flux in the optical light curves. A thin disk encounters difficulties here in explaining the required amount of intercepted flux. A twisted disk could be suggested here as a solution because its regions facing the center undergo increased illumination (Dubus et al. 1999; Esin et al. 2000), but the total benefit, calculated for the whole surface of one disk side, is ambiguous due to the effect of self-shielding (Suleimanov et al. 2007).

In the current study we prefer another explanation. We suggest the presence of matter above the disk in the form of a hot corona with temperature $\sim 2 \times 10^{6} \mathrm{~K}$ (e.g., corresponding to the atmosphere or the "warm corona" of Jimenez-Garate et al. 2002). By intercepting X-rays, the corona effectively increases the disk thickness. This idea manifests itself in the computation of the optical emission when we substitute the half-thickness of the disk $z_{0}$ by the value $z \approx(1.5-2) z_{0}$ representing the height of X-rays' interception. Furthermore, if the corona is inhomogeneous, i.e. consists of cooler blobs surrounded by hotter plasma, then multiple scattering between the blobs could explain the high values of the reprocessing efficiency (Suleimanov et al. 2003).

The outer parts of the disks in A 0620-00 and GRS 1124-68 have remarkably different properties. The disk in GRS 1124-68 turns out to be thinner and/or and weakly thermalizes the intercepted X-ray flux. Probably the illumination of the outer disk in A 0620-00 is more pronounced because of the higher Kerr parameter. If we freeze the disk thickness at a standard value and re-calculate models with the parameters used in Figs. 8 and 11, we find that the irradiating flux in A 0620-00 is twice that in GRS 1124-68. This could be the reason for the more developed corona above the outer disk in A 0620-00. Another possible source for the extended atmosphere of the disk is the magnetic pressure supporting the outer layers of the disk (e.g. Hirose et al. 2006). This would agree with the higher value of $\alpha$ in A $0620-00$, given that the magnetorotational instability generates the viscosity.

To bound the resulting parameters we need to invoke independent estimates of the mass and the distance. Then for A 0620-00 we obtain the intervals: $\alpha=0.7-0.95$ and the Kerr parameter $a=0.3-0.6$. For GRS $1124-68$ we find $\alpha=$ $0.55-0.75$ and the Kerr parameter $a \lesssim 0.4$ if the distance to the system lies between 3 and $4 \mathrm{kpc}$. If we do not apply the knowledge of the mass and the distance, we are nevertheless able to confirm the high value of the turbulent parameter: $\alpha \gtrsim 0.4$ (middle panels in Figs. 7 and 9). Such values of $\alpha$ are in aproximate agreement, but slightly higher than the commonly proposed values for thin, fully ionized disks: $\alpha \sim 0.1-0.4$ (e.g., King et al. 2007).

The accuracy of the estimates of $\alpha$ obtained suffers from limitations in the calculations of the X-ray flux made in the model and from the observational data. In this regard, we should also mention a simplified account of the tidal effects in the analytic model of a non-stationary disk (LS00). Presumably, the accretion disk in an X-ray nova binary has a fixed outer radius, at which a delta function-type transport of angular momentum to the orbital motion occurs due to tidal interactions. A physically more comprehensive consideration, involving a real form of the tidal torque (Papaloizou \& Pringle 1977; Smak 1984; Ichikawa \& Osaki 1994; Hameury \& Lasota 2005), could revise the solution (Eq. (1)) and thus change the estimates of $\alpha$ (see Eq. (19)). From this point of view, the value of $\alpha$ obtained for the outer disk is a "characteristic" parameter of the model. However, an advanced treatment would not change significantly the theoretical $\dot{M}$, and thus $\alpha$, if the tidal interactions are small in the whole disk and rise very steeply only near $R_{\text {out }}$ (Ichikawa \& Osaki 1994).

Our estimates of the Kerr parameter basically accord with a variety of estimates recently obtained for several stellar mass black holes: $a=0.77 \pm 0.05$ for M33 X-7, $a=0.65-0.75$ for GRO J1655-40, $a=0.75-0.85$ for $4 \mathrm{U} 1543-47, a=0.98-1.0$ for GRS 1915+105, $a<0.26$ for LMC X-3 (Liu et al. 2008; Shafee et al. 2006; McClintock et al. 2006; Davis et al. 2006). In these works, X-ray spectra are fitted by spectral models of a relativistic accretion disk (Li et al. 2005; Davis \& Hubeny 2006), and the black holes masses and orbital inclination angles are well known. Also, the Fe line method has been used to obtain an estimate for the black hole spin $a=0.86 \pm 0.05$ in GX 339-4 (Miller et al. 2008).

The model presented here can only be applied during the first few tens of days after the peak and fails to explain the late outburst phases. In the low/hard state, the thin disk model cannot adequately account for the observed spectra and energetics of X-ray novae. The ADAF model is believed to be appropriated instead (Esin et al. 1997, 2000). On the other hand, complex X-ray light curves at the early outburst phases, for example those of GRO J1655-40 and XTEJ1550-564, can hardly be interpreted by means of the present method. These binaries are highly inclined to the line of sight, and the flat thin disks are obscured by the outer thick disks during the early outburst (see Narayan \& McClintock 2005). The law of accretion rate evolution (Eq. (1)) also does not hold for the events involving an additional enhanced mass transfer from the secondary.

Our choice of the two X-ray novae stems from their exhibiting the most classical shape of X-ray nova light curves. The prospect of a future determination of $\alpha$ in ionized accretion disks in binary systems is likely to come from modeling the evolution of disk parameters during the time when the disk spectral component dominates in the spectrum of an exponentially decaying $\mathrm{X}$-ray nova in the high/soft state, and preferably with known parameters and orbital elements of the binary.

Acknowledgements. The X-ray data for A 0620-00 were kindly provided by E. Kuulkers. We thank R. Porkas, K. Postnov, and the anonymous referee for useful comments. The work was supported by the Russian Foundation for Basic Research (projects 06-02-16025 and 09-02-00032) and by the President's Program for Support of Leading Science Schools (grant Nsh.-4224.2008.2). V. F. Suleimanov is supported by the DFG grant SFB/Transregio 7 "Gravitational Wave Astronomy". N. I. Shakura is grateful to the Max Planck Institute for Astrophysics (Garching, Germany) for the opportunity for annual short-term study visits. G. V. Lipunova is grateful to the Offene Ganztagesschule of the Paul-Klee-Grundschule (Bonn, Germany) and Stadt Bonn for providing a possibility for her full-day scientific activity. 


\section{References}

Bardeen, J. M., \& Peterson, J. A. 1975, ApJ, 195, L65

Barret, D., McClintock, J. E., \& Grindlay, J. E. 1996, ApJ, 473, 963

Begelman, M. C., \& McKee, C. F. 1983, ApJ, 271, 89

Begelman, M. C., McKee, C. F., \& Shields, G. A. 1983, ApJ, 271, 70

Buff, J., Jernigan, G., Laufer, B., et al. 1977, ApJ, 212, 768

Cannizzo, J. K. 1993, The Limit Cycle Instability in Dwarf Nova Accretion Disks, Accretion Disks in Compact Stellar Systems, 6

Chandrasekhar, S. 1950, Radiative transfer (Oxford: Clarendon Press)

Chaty, S., Haswell, C. A., Malzac, J., et al. 2003, MNRAS, 346, 689

Chen, W., Shrader, C. R., \& Livio, M. 1997, ApJ, 491, 312

Cheng, F. H., Horne, K., Panagia, N., et al. 1992, ApJ, 397, 664

Cherepashchuk, A. M. 2000, Space Sci. Rev., 93, 473

Cunningham, C. T. 1975, ApJ, 202, 788

Davis, S. W., Blaes, O. M., Hubeny, I., \& Turner, N. J. 2005, ApJ, 621, 372

Davis, S. W., Done, C., \& Blaes, O. M. 2006, ApJ, 647, 525

Davis, S. W., \& Hubeny, I. 2006, ApJS, 164, 530

de Jong, J. A., van Paradijs, J., \& Augusteijn, T. 1996, A\&A, 314, 484

della Valle, M., Jarvis, B. J., \& West, R. M. 1991, Nature, 353, 50

Dubus, G., Lasota, J.-P., Hameury, J.-M., \& Charles, P. 1999, MNRAS, 303, 139

Dubus, G., Hameury, J.-M., \& Lasota, J.-P. 2001, A\&A, 373, 251

Duerbeck, H. W., \& Walter, K. 1976, A\&A, 48, 141

Ebisawa, K., Ogawa, M., Aoki, T., et al. 1994, PASJ, 46, 375

Eggleton, P. P. 1983, ApJ, 268, 368

El-Khoury, W., \& Wickramasinghe, D. 1999, MNRAS, 303, 380

Esin, A. A., Kuulkers, E., McClintock, J. E., \& Narayan, R. 2000, ApJ, 532, 1069

Esin, A. A., McClintock, J. E., \& Narayan, R. 1997, ApJ, 489, 865

Frank, J., King, A., \& Raine, D. J. 2002, Accretion Power in Astrophysics, 3rd edn. (Cambridge, UK: Cambridge University Press)

Gelino, D. M. 2004, in Rev. Mex. Astron. Astrofis. Conf. Ser., ed. G. Tovmassian, \& E. Sion, 20, 214

Gelino, D. M., Harrison, T. E., \& McNamara, B. J. 2001a, AJ, 122, 971

Gelino, D. M., Harrison, T. E., \& Orosz, J. A. 2001b, AJ, 122, 2668

Gilfanov, M., Churazov, E., \& Revnivtsev, M. 1999, A\&A, 352, 182

Hameury, J.-M., \& Lasota, J.-P. 2005, A\&A, 443, 283

Haswell, C. A., Robinson, E. L., Horne, K., Stiening, R. F., \& Abbott, T. M. C. 1993, ApJ, 411, 802

Hirose, S., Krolik, J. H., \& Stone, J. M. 2006, ApJ, 640, 901

Ichikawa, S., \& Osaki, Y. 1994, PASJ, 46, 621

Jimenez-Garate, M. A., Raymond, J. C., \& Liedahl, D. A. 2002, ApJ, 581, 1297

Kaluzienski, L. J., Holt, S. S., Boldt, E. A., \& Serlemitsos, P. J. 1977, ApJ, 212, 203

Ketsaris, N. A., \& Shakura, N. I. 1998, Astron. Astrophys. Transactions, 15, 193

Khruzina, T. S., Cherepashchuk, A. M., Bisikalo, D. V., Boyarchuk, A. A., \& Kuznetsov, O. A. 2003, Astron. Rep., 47, 621

King, A. R., \& Ritter, H. 1998, MNRAS, 293, L42

King, A. R., Pringle, J. E., \& Livio, M. 2007, MNRAS, 376, 1740

Ko, Y.-K., \& Kallman, T. R. 1994, ApJ, 431, 273

Kurucz, R. 1994, CD-ROMs Nos. 19-22, Cambridge, Mass.: Smithsonian Astrophysical Observatory

Kuulkers, E. 1998, New Astron. Rev., 42, 1

Lasota, J.-P. 2001, New Astron. Rev., 45, 449
Li, L.-X., Zimmerman, E. R., Narayan, R., \& McClintock, J. E. 2005, ApJS, 157, 335

Lipunova, G. V., \& Shakura, N. I. 2000, A\&A, 356, 363

Lipunova, G. V., \& Shakura, N. I. 2002, Astron. Rep., 46, 366

Lipunova, G. V., \& Suleimanov, V. F. 2004, Baltic Astron., 13, 167

Liu, J., McClintock, J., Narayan, R., Davis, S., \& Orosz, J. 2008, ArXiv e-prints, 803

Liutyi, V. M. 1976, Sov. Astron. Lett., 2, 43

Long, K. S., \& Kestenbaum, H. L. 1978, ApJ, 226, 271

Lyubarskij, Y. E., \& Shakura, N. I. 1987, Sov. Astron. Lett., 13, 386

McClintock, J. E., \& Remillard, R. A. 2003 [arXiv: astro-ph/0306213]

McClintock, J. E., Shafee, R., Narayan, R., et al. 2006, ApJ, 652, 518

Meyer, F., \& Meyer-Hofmeister, E. 1981, A\&A, 104, L10

Miller, K. J., \& Stone, J. M. 2008, ApJ, 534, 398

Miller, J. M., Reynolds, C. S., Fabian, A. C., et al. 2008, ArXiv e-prints, 802

Mitsuda, K., Inoue, H., Koyama, K., et al. 1984, PASJ, 36, 741

Morrison, R., \& McCammon, D. 1983, ApJ, 270, 119

Narayan, R., \& McClintock, J. F. 2005, ApJ, 623, 1017

Narayan, R., Mahadevan, R., \& Quataert, E. 1998, in Theory of Black Hole Accretion Disks, 148

Orosz, J. A., Bailyn, C. D., McClintock, J. E., \& Remillard, R. A. 1996, ApJ, 468,380

Paczynski, B. 1977, ApJ, 216, 822

Page, D. N., \& Thorne, K. S. 1974, ApJ, 191, 499

Papaloizou, J., \& Pringle, J. E. 1977, MNRAS, 181, 441

Proga, D., \& Kallman, T. R. 2002, ApJ, 565, 455

Riffert, H., \& Herold, H. 1995, ApJ, 450, 508

Różańska, A., \& Czerny, B. 1996, Acta Astron., 46, 233

Shafee, R., McClintock, J. E., Narayan, R., et al. 2006, ApJ, 636, L113

Shahbaz, T., Naylor, T., \& Charles, P. A. 1994, MNRAS, 268, 756

Shahbaz, T., Naylor, T., \& Charles, P. A. 1997, MNRAS, 285, 607

Shakura, N. I., \& Sunyaev, R. A. 1973, A\&A, 24, 337

Shimura, T., \& Takahara, F. 1995, ApJ, 445, 780

Shrader, C. R., \& Gonzalez-Riestra, R. 1993, A\&A, 276, 373

Smak, J. 1984, Acta Astron., 34, 161

Sobolev, V. V. 1949, Uchenye Zapiski Leningrad. Univ., Seria Matem. Nauk, 18, N 1163

Sobolev, V. V. 1969, Course in Theoretical Astrophysics (NASA), 1, F-531

Speith, R., Riffert, H., \& Ruder, H. 1995, Comput. Phys. Comm., 88, 109

Suleimanov, V., Meyer, F., \& Meyer-Hofmeister, E. 1999, A\&A, 350, 63

Suleimanov, V., Meyer, F., \& Meyer-Hofmeister, E. 2003, A\&A, 401, 1009

Suleimanov, V. F. 1991, Sov. Astron. Lett., 17, 245

Suleimanov, V. F., Lipunova, G. V., \& Shakura, N. I. 2007, Astron. Rep., 51, 549

Sulkanen, M. E., Brasure, L. W., \& Patterson, J. 1981, ApJ, 244, 579

Tanaka, Y., \& Shibazaki, N. 1996, ARA\&A, 34, 607

Turner, N. J. 2004, ApJ, 605, L45

van den Bergh, S. 1976, AJ, 81, 104

van Paradijs, J. 1996, ApJ, 464, L139

Weaver, H., \& Williams, D. R. W. 1974, A\&AS, 17, 1

Wu, C.-C., Panek, R. J., Holm, A. V., Schmitz, M., \& Swank, J. H. 1983, PASP, 95,391

Zombeck, M. V. 1990, Handbook of space astronomy and astrophysics (Cambridge: University Press), 2nd edn. 